\title{
Diagnostic Value of T SPOT TB in Infant to 5-Year Old Children with Primary Forms of Tuberculosis
}

\author{
Svetlana Atanasova Velizarova \\ Department of Pulmonary Diseases, Medical University, Sofia 1432, Bulgaria
}

\begin{abstract}
IGRA (interferon gamma release assay) testing has marked a new start in the diagnosis of tuberculosis. There is still, however, discrepant evidence in terms of its effectiveness in the early childhood. The purpose of this study was to compare the effectiveness of the new T SPOT TB (ELISPOT TB test) and the TST (tuberculin skin test) in infant children with active tuberculosis. Fifty one (51) children, who were treated at the University Children's Clinic for Pulmonary Diseases in 2009 were studied. The average age of the children was $2.15 \pm 1.39$ years. Upon examination with the TST, only 2 children reacted negatively, $45.1 \%$ were with size of the infiltrate $6 \mathrm{~mm}$ to $14 \mathrm{~mm}$ at the $72 \mathrm{nd} \mathrm{h}$ and $51.0 \%$ were with size of the infiltrate larger than $15 \mathrm{~mm}$. Upon examination with the T SPOT TB, $69.6 \%$ of the children gave positive results, which was significantly less than the $96.1 \%$ positive results, obtained with the TST, and in consistency with the study results of other authors. By using both tests, the high rate of $76.7 \%$ of positive results was achieved. The concordance between TST and T SPOT TB was $76.4 \%$. This gives us reason to believe that despite the difficulty of diagnosing tuberculosis in infancy, the use of both tests increases our diagnostic capabilities.
\end{abstract}

Key words: T SPOT TB, TST, tuberculosis in children.

\section{Introduction}

Tuberculosis is a disease that undergoes a renaissance in recent years. One third of the world population is infected. Over 8 million get sick each year and 2-3 million die from tuberculosis.

In the European region, 450,000 new cases were detected and 69,000 people died only in 2009. In Europe, 50 people are at a risk and 8 die every hour from tuberculosis. $15 \%$ of the patients are resistant to tuberculostatics.

There is a big difference between the incidence of tuberculosis in Eastern and Western Europe-from 4/100,000 in Switzerland to 177/100,000 in Turkey. In Bulgaria and Romania, the incidence is $53 / 100,000$, respectively.

New diagnostic methods for the early diagnosis of tuberculosis are constantly searched for. It was necessary to identify antigens that are highly specific to

Corresponding author: Svetlana Atanasova Velizarova, M.D., research fields: immunology, tuberculosis, BCG vaccination and pulmonary diseases in childhood. E-mail: velizarovasv@gmail.com,sv_velizarova@abv.bg. the tuberculosis bacterium and were not presented in the BCG (Bacille Calmette-Guerin) vaccine or atypical mycobacteria. This became possible after reading the genome of the tuberculosis bacterium. These specific antigens are ESAT-6 (early secreted antigenic target 6) and CFP-10 (culture filtrate protein 10). These antigens are localised in the gene, differentiated as the region of deletion 1 (RD 1) segment of Mycobacterium tuberculosis. Both proteins have been associated with the virulence and cytotoxicity of the tuberculosis bacterium. These proteins are directly responsible for the specific T-cell immune response.

The T SPOT TB was found by Ajit Lalvani in 2001 and was first presented at the symposium in Paris in 2004. This test counts directly the interferon-gamma producing effector cells. Separated lymphocytes are placed into wells with antigens and the specific antigens are affixed to the interferon-gamma producing T-cells.

The objective of this study was to compare the effectiveness of the T SPOT TB and the TST in children suffering from tuberculosis in infancy. 


\section{Materials and Methods}

\subsection{Study Participants}

The study was conducted at the University Children's Clinic of Pulmonary Diseases in Sofia, from January to December 2009, in children with active tuberculosis. The children were 0 to 5 years of age.

\subsection{Inclusion Criteria}

Tuberculosis was diagnosed, based on:

(1) signs and symptoms of active tuberculosis;

(2) microbiological and culture confirmation;

(3) abnormal radiograms;

(4) history of contacts with actively diseased adults.

As a result of meeting all these criteria, only children with diagnosed tuberculosis were studied, prior to initiating anti-tuberculosis therapy.

\subsection{Screening for Active Tuberculosis}

(1) Gastric lavage was taken from all children and were examined for tuberculosis bacteria directly and on a culture;

(2) The Mantoux tuberculin test was performed on the left forearm, strictly intradermally, with 5 IU of the Bulgarian PPD tuberculin. The results were read at the 72nd $\mathrm{h}$. The results were classified into three groups, depending on the size of the infiltrate: first group with negative results $(0-5 \mathrm{~mm})$, second group (6-14 mm) and third group (larger than $15 \mathrm{~mm}$ );

(3) The presence or absence of a BCG scar on the left shoulder was recorded in all children (in Bulgaria, compulsory BCG vaccination has been carried out since 1960).

\subsection{Laboratory Analyses}

The $\mathrm{T}$ SPOT $\mathrm{TB}$ was tested in the Central Immunological Laboratory, Alexandrovska Hospital, in accordance with the recommendations of Oxford Immunotec, Abingdon, UK. The blood was collected in $4 \mathrm{~mL}$ tubes and PBMCs (peripheral blood mononuclear cells) were prepared within $2 \mathrm{~h}$, following standard procedures. All procedures, including the result interpretations, were performed in accordance with the recommendations of the Manufacturer. The values of the spot forming cells were counted by using a microscope. The results were evaluated in accordance with the standards of Oxford Immunotec.

\subsection{Statistical Analysis}

The statistical analysis was performed by using the SPSS 15. The failed or indefinite results were not included in the analysis. The rate ratio for both tests was examined in various forms of tuberculosis and different diagnostic criteria. The concordance and kappa were examined.

\section{Results and Discussion}

All children were hospitalised for treatment at the clinic, referred by the general practitioners or district dispensaries. All children were of Bulgarian nationality, aged from 0 to 5 years. The duration of the study was 12 months. Girls and boys were 25 and 26 children, respectively.

The group of children with tuberculosis of the tracheobronchial lymph nodes (37 children) was the largest, followed by these of the children with primary tuberculous complex (9 children), extrapulmonary tuberculosis (3 children) and haematogenous disseminated forms (2 children), which is not much different from the structure of morbidity in Bulgaria for this age group (Fig. 1).

All children were screened for the presence or absence of a BCG vaccination scar. In 33 children, there was no BCG scar, which is associated either with the late appearance of the scar in children under 1 year of age or omissions in our vaccination programme. Since the presence of a scar is the only criterium for exact performance of the BCG vaccination, we will focus on the monitoring of these children, in terms of severity of the disease.

Considering the course of the TB disease and its clinical manifestations, a large number of the children 
(41 children) were admitted to the clinic after detection of an adult with bacteriological confirmation.

Based on the aetiopathogenesis of tuberculosis in the childhood, it is known that a very small percentage of children are bacteriological confirmation. In this age group, 25.5\% were BK (Bacillus Koch)-positive directly or on a culture, which is much higher than the average $14 \%$ for the child age.

It is noteworthy that in this age group, only two children reacted negatively to the TST with 5 IU of PPD tuberculin. $45.1 \%$ had size of the infiltrate $6 \mathrm{~mm}$ to $14 \mathrm{~mm}$ at the $72 \mathrm{nd} \mathrm{h}$ and $51.0 \%$ were with tuberculin reactions larger than $15 \mathrm{~mm}$ (Fig. 2).

Upon the examination with the T SPOT TB, 69.6\% of the children gave positive results, as 5.9\% responded to ESAT-6, $15.7 \%$ to CFP and $47.1 \%$ to both antigens (Fig. 3).
The average age of the children studied was $2.15 \pm$ 1.39 years. The mean values of the tuberculin reaction were $13.67 \pm 4.7 \mathrm{~mm}$. As demonstrated by our statistics, the average size of the infiltrate was within the normal range, but in most of the children, the quality characteristics were typical for a virulent nature of the infiltrate, with presented bullae, vesicles, petechiae, uneven borders of the infiltrate, residual pigmentation or crusts. This shows how important the interpretation of the tuberculin skin test is.

The mean values of the ESAT- 6 were $14.73 \pm 14.22$ spots and the mean values of CFP $10-18.69 \pm 16.52$ spots. It can be seen that the number of spots, reacted to CFP 10, is higher by almost 4 points, which is consistent with the publications considering this antigen as the antigen of the early phase of tuberculous infection (Fig. 4).

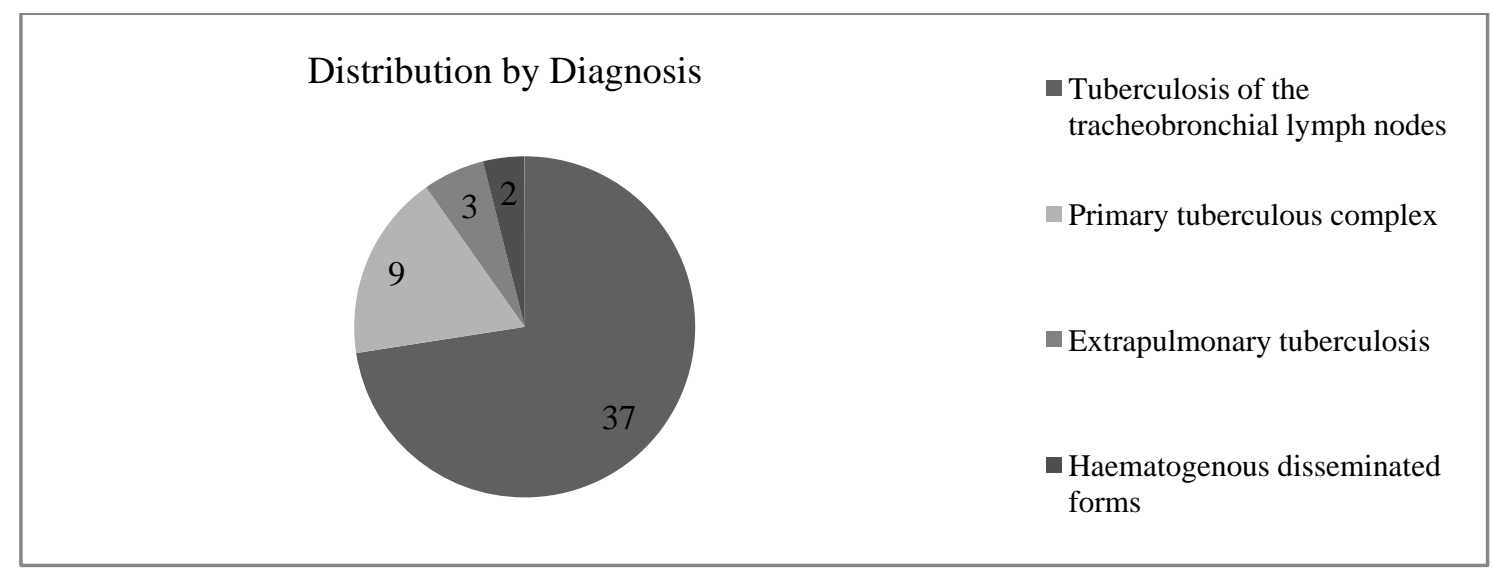

Fig. 1 Distribution by diagnosis.

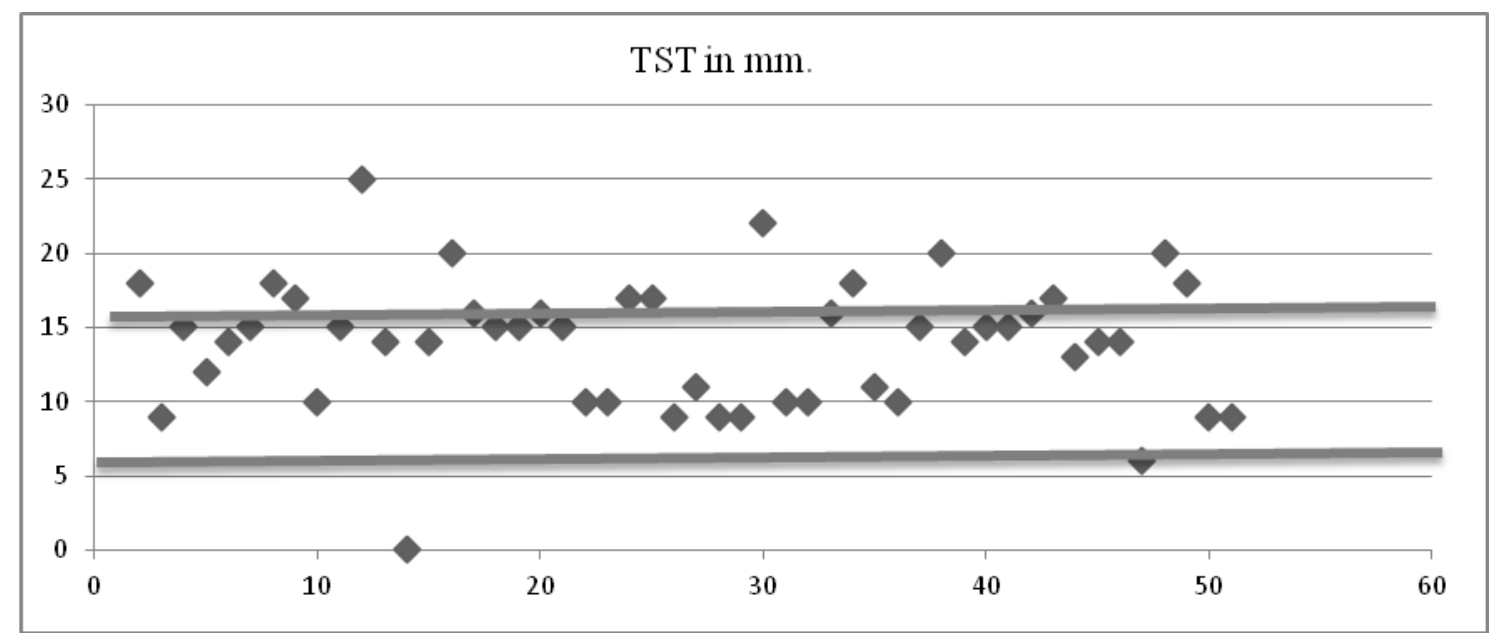

Fig. 2 TST reactions at the 72nd h (5 IU of PPD tuberculin). 


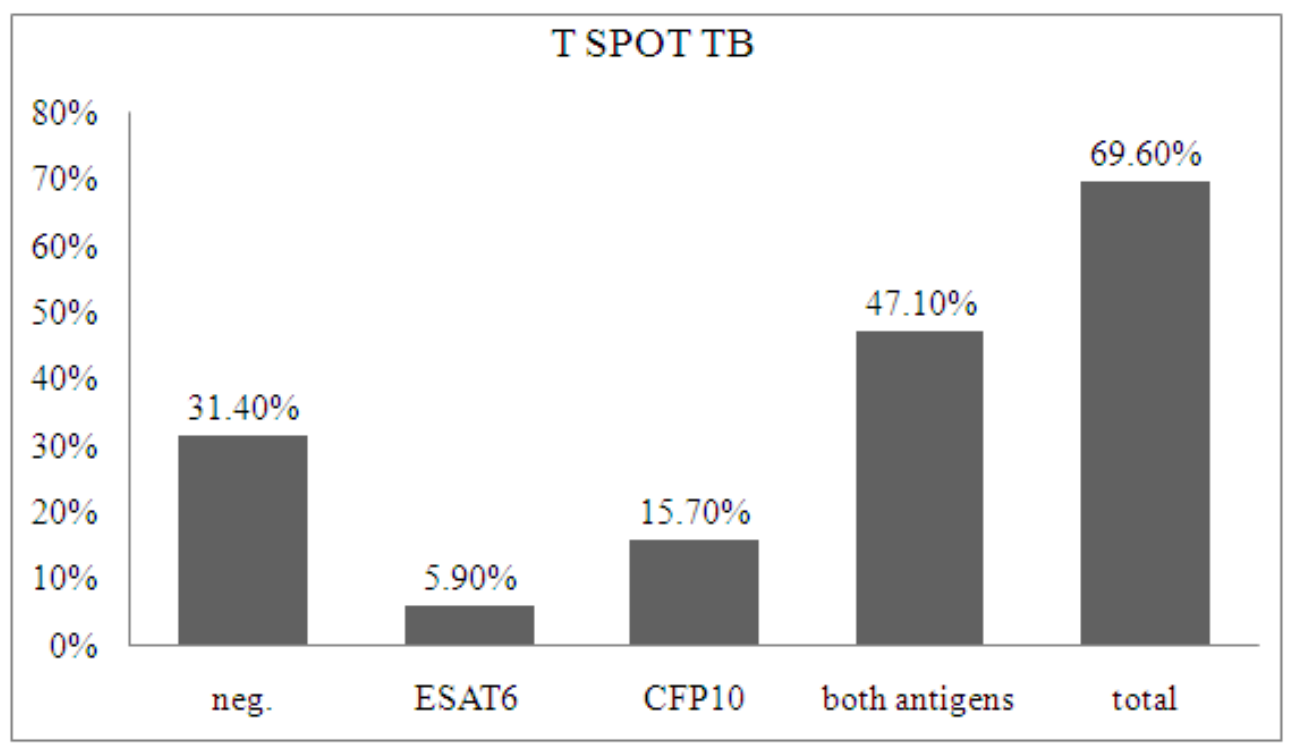

Fig. 3 Effects of T SPOT TB rates.

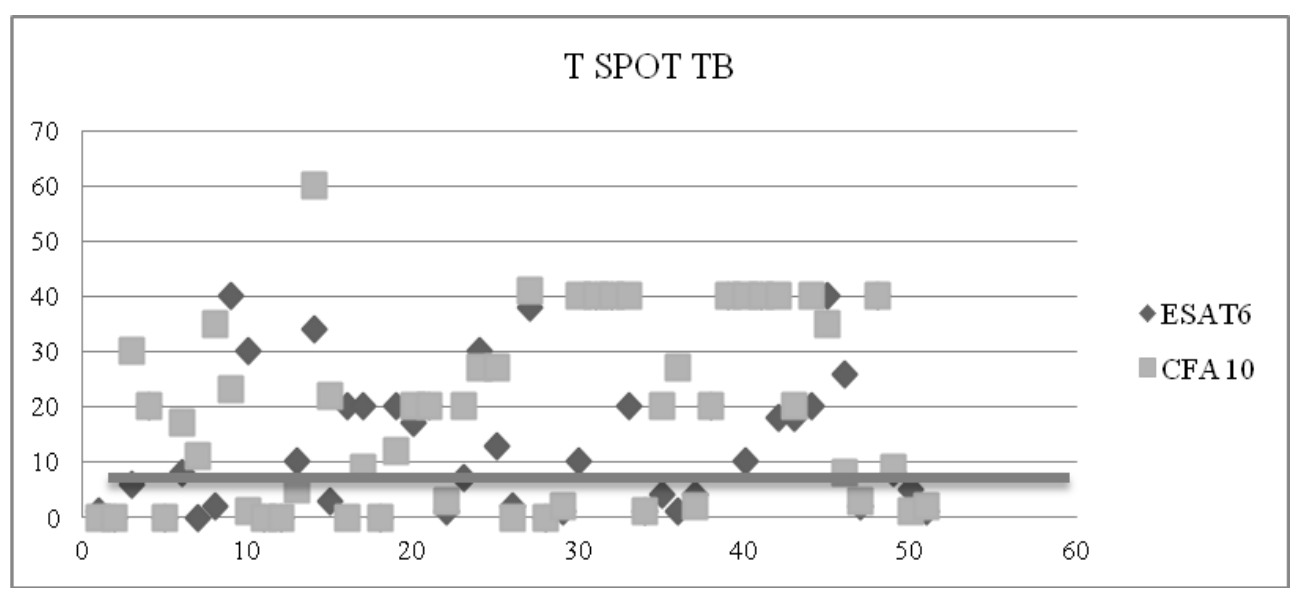

Fig. 4 T SPOT TB reaction in spots.

The TST and the T SPOT TB in the different forms of tuberculosis were monitored.

In tuberculosis of the tracheobronchial lymph nodes, 22 children were with TST $>15 \mathrm{~mm}$ and 14 with TST of 6-14 mm. Twenty six (26) of the children responded positively to the T SPOT TB, as 7 of them to CFP 10 and 18 to both antigens. This once again supports the above stated [1-3] (Fig. 5).

In the primary tuberculosis complex group, 5 children were with normergic TSTs, but with signs of virulence, and 4 children were with hyperergic TSTs. For this nosological entity, the $\mathrm{T}$ SPOT TB was positive in 7 patients, as there was 1 responder to ESAT-6, 1 to CFP 10 and 5 responders to both antigens, which is in support of the aetiopathogenesis of the primary complex, where a large and continuous invasion of tuberculosis bacterium is required.

The children with haematogenous disseminated forms and tuberculous meningitis were with normergic tuberculin reactions and negative T SPOT TB reactions, which can be explained, on the one hand, by the severity of the disease and on the other, with the young age of the children and the insufficient amount of interferon-gamma producing effector T-lymphocytes [4-7].

The presence or absence of a BCG scar did not affect the reaction to tuberculin. Both groups responded equally, with norergic and hyperergic tuberculin 
reactions. The group of children without scars (33 children) was predominant, which once again raises the question of the need of control over the vaccination programme.

For the T SPOT TB, $67.7 \%$ of the children with no BCG scars reacted positively; $6.1 \%$ of them to ESAT-6, $15.2 \%$ to CFP 10 and $45.5 \%$ to both antigens. In the children with BCG scars, $73.2 \%$ were positive: $5.6 \%$ to ESAT-6, $16.7 \%$ to CFP 10 and $50.0 \%$ to both antigens, respectively. BCG vaccination has no effect on $\mathrm{T}$ SPOT TB, since it lacks the antigens of BCG strains.

In Fig. 6, it can be seen that the children, who were in contact with tuberculous adults, have pronounced reactions to both the TST and the T SPOT TB, which once again shows that the close contact with a tuberculous patient affects the immunological reactivity of the body and results in more frequently in a disease [8-10, 12].
It is interesting to note that in the BK-positive children, the rate of hyperergia was only $30.8 \%$, while in the BK-negative children, the hyperergia was $57.9 \%$. In the BK-positive children, only $38.5 \%$ were positive to the T SPOT TB, indicating that in this age group, the diagnostic capabilities of the tuberculin test are greater (Fig. 7).

As a result, we determined the sensitivity in TST for the cases with sizes of the infiltrate larger than $10 \mathrm{~mm}$, because we found that in the majority of the children, the tuberculin reaction was larger than $10 \mathrm{~mm}$, with quality characteristics of virulence [12-14].

In sizes of the infiltrate larger than $10 \mathrm{~mm}$, the sensitivity was 61.5\%, which corresponds to the diagnostic capabilities of the test, where both quantitative and qualitative indicators of virulence are taken into account. In sizes of the infiltrate larger than $10 \mathrm{~mm}$, sensitivity of T SPOT TB was 38.5\%.

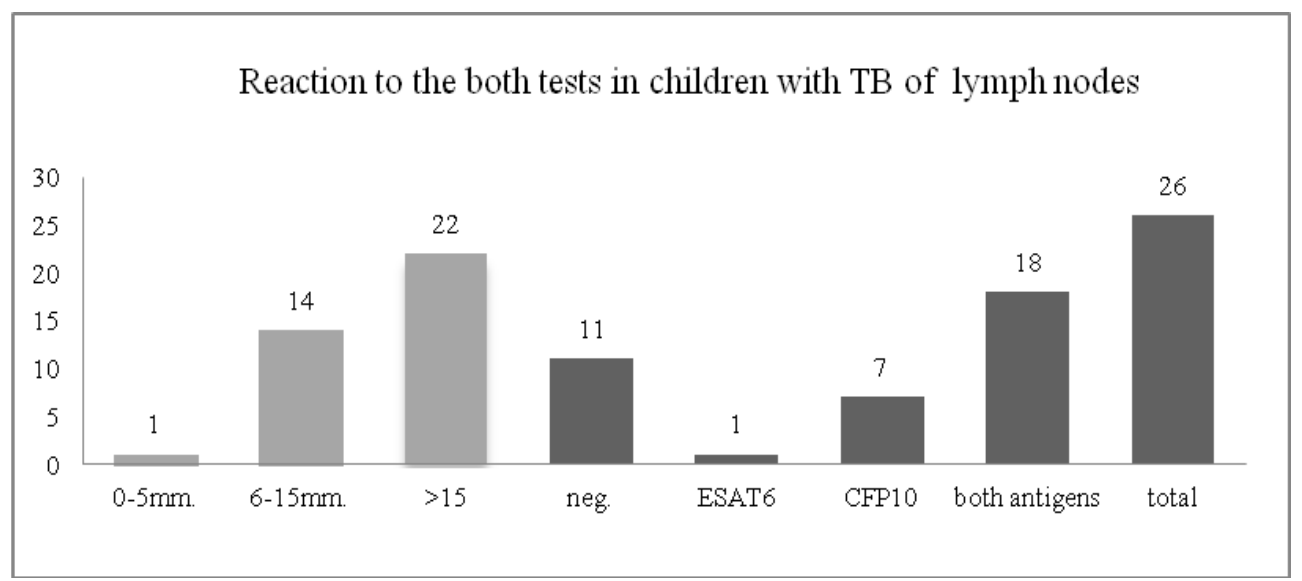

Fig. 5 Reaction to both tests in children with TB lymph nodes.

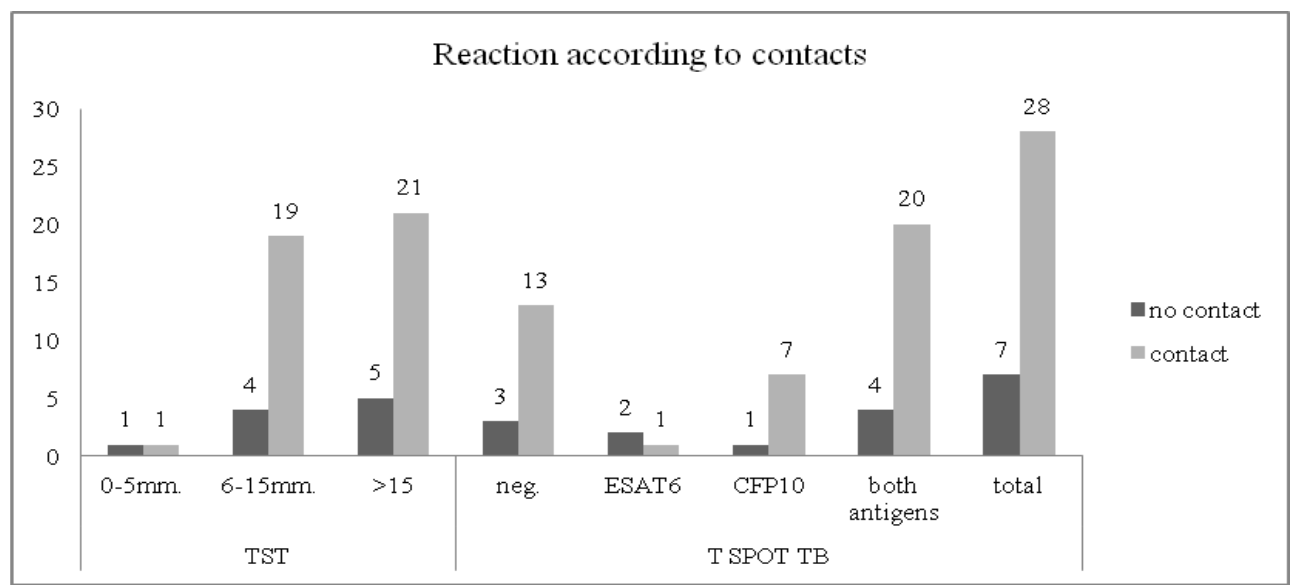

Fig. 6 Reaction to both tests in children with contacts to MTB positive adults. 


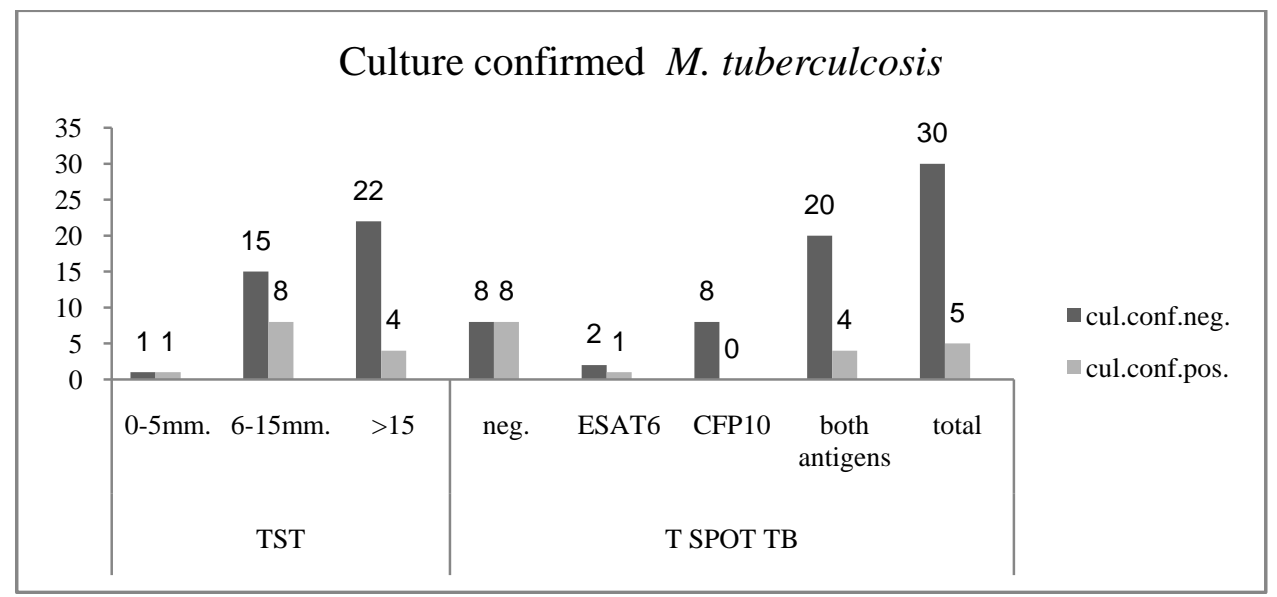

Fig. 7 Reaction to both tests in children with culture confirmed M. tuberculosis.

It was of crucial importance for us to examine whether and what type of relationship is available between the two tests. For achieving these objectives, we used the concordance and kappa.

In the children of up to 5 years of age, we found a concordance of $76.4 \%$ at a likelihood ratio of 7.81-df1- $P<0.005$ and kappa 0.368 at $P=0.04$. This suggests a relatively good correlation between the two immunological tests.

The diagnosis of active tuberculosis in children remains a challenge in pediatrics, since the bacteriological confirmation is the exception rather than the rule. In most cases, the diagnosis is based on the symptoms, history of exposure, radiographic changes and evidence of BCG vaccination.

In this study, we made an attempt to investigate the diagnostic potential of the T SPOT TB in children with active tuberculosis, which showed less sensitivity, compared to adults. Many authors believe that in a primary infection, such as the case in small children, due to the specificity of the immunological response, there is less production of interferon-gamma by the effector cells in the peripheral blood, while in reactivation of the infection in adults, there is significantly more specific effector cells and therefore the $\mathrm{T}$ SPOT TB sensitivity is higher. Many studies have shown that a certain amount of antigen-specific effector cells in the peripheral blood is required, in order to obtain adequate results in the T SPOT TB test.
In some cases, the initially negative T SPOT TB results become positive in the process of monitoring, which supports the above hypothesis [15-17].

According to the results obtained, the negative $\mathrm{T}$ SPOT TB does not exclude an active tuberculosis, particularly in children of up to 5 years of age. However, an examination with the $\mathrm{T}$ SPOT TB, especially in countries with compulsory BCG vaccination, may contribute to the overall diagnostic process, especially in combination with the TST. In the event of a negative T SPOT TB but other diagnostic criteria presented for active tuberculosis, tuberculostatic therapy should be initiated. This is particularly important for children of up to 5 years of age and complies with the recommendations of ECDC Guidance-Use of interferon-gamma release assays in support of TB diagnosis Stockholm, 2011 [18-21].

This gives us a reason to believe that despite the difficulty of diagnosing tuberculosis in this age group, by using both tests we can achieve $76.7 \%$ positive results. Regardless of the complex immunological micro-macroorganism relationships in the early childhood, both immunological tests have their value. Of course, in children with negative T SPOT TB tests and other diagnostic criteria presented, the treatment should be started.

\section{Conclusions}

From the above-stated, we can draw the following 
conclusions:

(1) T SPOT TB has a lower diagnostic capability than TST in the most common form of primary tuberculosis-tuberculosis of the tracheobronchial lymph nodes;

(2) The T SPOT TB reveals its highest diagnostic capabilities in the primary tuberculosis complex;

(3) In the severe forms of tuberculosis, such as tuberculous meningitis and haematogenous disseminated tuberculosis, the diagnostic capabilities of both tests are low, due to the severe immunosuppression in young children;

(4) Upon the use of both tests, we can achieve the high rate of $76.7 \%$ of positive results;

(5) The concordance between TST and T SPOT TB was $76.4 \%$, indicating high diagnostic capabilities of the use of both tests.

\section{Acknowledgments}

The study was funded by the Medical University-Sofia under Grant Contract No. 2/ 15/07/2008.

\section{References}

[1] S. Andronikou, E. Joseph, S. Lucas, S. Brachmeyer, G. Du Toit, H. Zar, et al., CT scanning for the detection of tuberculous mediastinal and hilar lymphadenopathy in children, Pediatr. Radiol. 34 (3) (2004) 232-236.

[2] P. Andersen, M.E. Munk, J.M. Pollock, T.M. Doherty, Specific immune-based diagnosis of tuberculosis, Lancet 356 (9235) (2000) 1099-1104.

[3] A.T. Cruz, A.M. Geltemeyer, J.R. Starke, J.A. Flores, E.A. Graviss, K.C. Smith, Comparing the tuberculin skin test and T-SPOT.TB blood test in children, Pediatrics 127 (1) (2011) e31-e38.

[4] P. Beffa, A. Zellweger, J.P. Janssens, P. Wrighton-Smith, J.P. Zellweger, Indeterminate test results of T-SPOT.TB performed under routine field conditions, Eur. Respir. J. 31 (4) (2007) 842-846.

[5] B.J. Marais, R.P. Gie, H.S. Schaaf, N. Beyers, P.R. Donald, J.R. Starke, Childhood pulmonary tuberculosis: Old wisdom and new challenges, American Journal of Respiratory and Critical Care Medicine 173 (10) (2006) 1078-1090.

[6] L. Bianchi, L. Galli, M. Moriondo, G. Veneruso, L. Becciolini, C. Azzari, et al., Interferon-gamma release assay improves the diagnosis of tuberculosis in children, Pediatric Infectious Disease Journal 28 (6) (2009) 510-514.

[7] G.H. Bothamley, L. Ditiu, G.B. Migliori, C. Lange, TBNET contributors, Active case finding of tuberculosis in Europe: A Tuberculosis Network European Trials Group (TBNET) survey, Eur. Respir. J. 32 (4) (2008) 1023-1030.

[8] T.G. Connell, N. Ritz, G.A. Paxton, J.P. Buttery, N. Curtis, S.C. Ranganathan, A three-way comparison of tuberculin skin testing, QuantiFERON-TB gold and T-SPOT.TB in children, PLoS ONE 3 (7) (2008) e2624.

[9] P.M. Enarson, D.A. Enarson, R. Gie, Management of tuberculosis in children in low-income countries, Int. J. Tuberc. Lung Dis. 9 (12) (2006) 1299-1304.

[10] B. Kampmann, E. Whittaker, A. Williams, S. Walters, A. Gordon, N. Martinez-Alier, et al., Interferon- $\gamma$ release assays do not identify more children with active tuberculosis than the tuberculin skin test, Eur. Respir. J. 33 (6) (2009) 1374-1382.

[11] A. Lalvani, L. Richeldi, H. Kunst, Interferon gamma assays for tuberculosis, Lancet Infect. Dis. 5 (6) (2005) 322-324.

[12] A.M. Mandalakas, A.K. Detjen, A.C. Hesseling, A. Benedetti, D. Menzies, Interferon-gamma release assays and childhood tuberculosis: Systematic review and meta-analysis, International Journal of Tuberculosis and Lung Disease 15 (8) (2011) 1018-1032.

[13] M.P. Nicol, R.J. Wilkinson, The clinical consequences of strain diversity in Mycobacterium tuberculosis, Trans. R. Soc. Trop. Med. Hyg. 102 (10) (2008) 955-965.

[14] M.P. Nicol, B. Kampmann, P. Lawrence, K. Wood, S. Pienaar, D. Pienaar, et al., Enhanced anti-mycobacterial immunity in children with erythema nodosum and a positive tuberculin skin test, J. Invest. Dermatol. 127 (9) (2007) 2152-2157.

[15] M.P. Nicol, R.J. Wilkinson, Evidence guiding the treatment of children with mycobacterial diseases, Clin. Infect. Dis. 44 (8) (2007) 1065-1066.

[16] M.P. Nicol, D. Pienaar, K. Wood, B. Eley, R.J. Wilkinson, H. Henderson, et al., Enzyme-linked immunospot assay responses to early secretory antigenic target 6 , culture filtrate protein 10 , and purified protein derivative among children with tuberculosis: Implications for diagnosis and monitoring of therapy, Clin. Infect. Dis. 40 (9) (2005) 1301-1308.

[17] M. Pai, R. Joshi, M. Bandyopadhyay, P. Narang, S. Dogra, B. Taksande, et al., Sensitivity of a whole-blood interferon-gamma assay among patients with pulmonary tuberculosis and variations in $\mathrm{T}$ cell responses during anti-tuberculosis treatment, Infection 35 (2) (2007) 98-103. 
[18] J.R. Starke, Pediatric tuberculosis: Time for a new approach, Tuberculosis (Edinb) 83 (1-3) (2003) 208-212.

[19] V. Bosshard, P. Roux-Lombard, T. Perneger, M. Metzger, R. Vivien, T. Rochat, et al., Do results of the T-SPOT.TB interferon- $\gamma$ release assay change after treatment of tuberculosis?, Respiratory Medicine 103 (1) (2009) 30-34

[20] ECDC TB Team European Centre for Disease Prevention and Control, Stockholm, 18 March 2011 Three key messages on childhood tuberculosis. http://www.cdc.gov/tb/publications/newsletters/notes/TB N_2_11/images\%5Ctbn211.pdf (accessed Mar. 28, 2011).

[21] ECDC Guidance, Use of Interferon-Gamma Release Assays in Support of TB Diagnosis, Stockholm, 2011, http://ecdc.europa.eu/en/publications/publications/1103_g ui_igra.pdf (accessed Nov. 2, 2011). 